\title{
Mapping the physics research space: a machine learning approach
}

\author{
Matteo Chinazzi ${ }^{*}$ (D), Bruno Gonçalves², Qian Zhang ${ }^{1}$ and Alessandro Vespignani ${ }^{1}$
}

"Correspondence:
m.chinazzi@northeastern.edu
${ }^{1}$ MOBS Lab, Northeastern
University, Boston, USA
Full list of author information is
available at the end of the article

\begin{abstract}
Scientific discoveries do not occur in vacuum but rather by connecting existing pieces of knowledge in new and creative ways. Mapping the relation and structure of scientific knowledge is therefore central to our understanding of the dynamics of scientific production. Here we introduce a new approach to generate scientific knowledge maps based on a machine learning approach that, starting from the observed publication patterns of authors, generates an $\mathrm{N}$-dimensional space where it is possible to measure the similarity or distance between different research topics and knowledge domains. We provide an implementation of the proposed approach that considers the American Physical Society publications database and generates a map of the research space in Physics that characterizes the relation among research topics over time. We use this map to measure two indicators, the research capacity fingerprint and the knowledge density, to profile the research activity in physical sciences of more than 400 urban areas across the world. We show that these indicators can be used to analyze and predict the evolution over time of the research capacity and specialization of specific geographical areas. Furthermore we provide an extensive analysis of the relation between socio-economic development indicators and the ability to produce new knowledge for 67 countries, as measured by our approach, highlighting some key correlates of scientific production capacity. The proposed approach is scalable to very large datasets and can be extended to study other disciplines and research areas without having to rely on ad-hoc science classification schemes.
\end{abstract}

Keywords: Embeddings; Research space; Principle of relatedness; Fingerprinting scientific production; Revealed comparative advantage; Predicting research specialization

\section{Main text}

The definition of meaningful maps of the research space is a fundamental step in the study of the emergence of scientific areas and the characterization of the drivers of knowledge production and consumption. Mapping the relation and structure of scientific knowledge is indeed one of the key elements towards the understanding of the dynamics of science and has practical applications in the information retrieval and classification of the ever growing output of the research community. The recent abundance of large scale bibliographic datasets has provided momentum to the study of the dynamics and structure of science $[1,2]$. Studies have shown that it is possible to characterize the evolution of entire disciplinary areas [3, 4], identify general trends in science [5-7], characterize the effect

(c) The Author(s) 2019. This article is distributed under the terms of the Creative Commons Attribution 4.0 International License (http://creativecommons.org/licenses/by/4.0/), which permits unrestricted use, distribution, and reproduction in any medium, provided you give appropriate credit to the original author(s) and the source, provide a link to the Creative Commons license, and indicate if changes were made. 
of memory and attention [8-10], and measure the emergence and relevance of interdisciplinary efforts [11-14]. Considerable progress has been made also in the study of the mobility of researchers both in space and among research topics [15-22] and in the characterization and explanation of collaboration dynamics between individual researchers, institutions, and countries [23-34]. Remarkably, the advances in the field have opened the path to the understanding and prediction of scientific productivity and individual researchers' careers [35-41]. In this context, the visualization and mapping of the research space is a major tool in the study of the scientific portfolio of authors, institutions, and countries, the co-production of bibliographic items, and the quantitative characterization of similarity between scientific topics [3, 4, 42-47].

One of the hurdles in defining large-scale knowledge maps is that the approaches proposed in the literature typically rely on well defined scientific taxonomies. Indeed, it has been shown that it takes a non-trivial effort to analyze the evolution of trending topics in science when only keywords are used rather than well established classification schemes [5]. Here, we propose a new methodology that uses recent developments stemming from the Natural Language Processing (NLP) machine learning literature on word embeddings [48-54] to create a spatial mapping of the research space of scientific disciplines. In this space, scientific topics are represented using $N$-dimensional vectors, and the similarity among topics can be organically computed as their spatial closeness in this $N$-dimension space. We apply our methodology to articles published in American Physical Society's (APS) journals in the period 1986-2009 to generate a map of the physics research space, and show that our method correctly reproduces the scientific structure encoded within the Physics and Astronomy Classification Scheme (PACS). Indeed, while the research topics are labeled according to the latter, our method does not use the hierarchical structure embedded into the PACS scheme to learn the physics research space. Rather, we use the structure of the classification scheme as a ground truth to validate our findings, showing that our methodology does not require the a-priori existence of a well-defined scientific or technical taxonomy to produce a meaningful knowledge space.

In order to show the potential of our approach, we project the physical sciences research activity of different geographical units (urban areas and countries) in the research space obtained with our methodology, fingerprinting the scientific expertise of entire geographical areas. The scientific fingerprint encodes the scientific production and allows to visually characterize the specialization into topical areas of each geographical entity. Using the scientific expertise fingerprint, we can appropriately define and measure a knowledge density indicator that characterizes the research capacity of each specific geographical unit. We show that the knowledge density is a good predictor of the future probability of an urban area to become a specialized scientific producer in a specific research topic in Physics. The countries' knowledge density is also significantly related to the overall level of economic and technical development of nations, as shown by its association with the development indicators of 67 countries, hinting to the possibility of using the research space to explain the patterns of socio-economic and technical development of countries. In other words, the present work shows that machine learning techniques can be used not only to advance the study of the structure and dynamics of scientific knowledge but also to systematically generate new indicators that can measure the overall level of scientific capabilities of nations. While a specific physics literature database is used to exemplify our approach, the presented methodology can be applied and generalized to other schol- 
arly/bibliometric databases, thus opening the path to the definition of knowledge maps not relying on pre-defined taxonomies or classification schemes.

\section{Results}

The core assumption behind our approach is that research topics can be simply characterized by sets of keywords extracted from individual publications, patents, and other scientific artifacts. The relations among scientific areas are generally provided by measures of similarity among the research topics, inferred from co-occurrences of keywords in papers, citations, or other bibliographic indicators. Common approaches range from co-word similarity, to citation linkages, or more sophisticated vector space models [42, $55,56]$.

In our case, to extract the labels that we are going to use to identify the research topics, we consider all the articles published in American Physical Society's (APS) journals in the period 1986-2009 and we associate to each article: (a) a set of authors; and (b) a set of research topics identified using the Physics and Astronomy Classification Scheme (PACS) codes reported in each publication. Given this data, we select the scientific output of each author by keeping track of all the research topics, identified by the PACS codes, in which she/he has published on in a given time window. Then, we represent each scientist as a bag-of-topics and we use this information to train our embedding model and recover the vector embeddings for each PACS code (as shown in Fig. 1). In the machine learning literature, supervised and unsupervised vector space models have been used to perform exactly this task: embed words in a high dimensional space in which semantically similar words are mapped into neighboring points. Here, we use the general-purpose embedding approach proposed by [54] to map research topics (i.e. PACS codes) into a research space in which scientifically similar topics are placed close to each other. The motivation behind this methodology lies in the principle of relatedness $[57,58]$ : i.e. it is easier to specialize and work in related research areas requiring a set of common skills/knowledge. Each individual is thus assumed to have a given set of skills/knowledge which allow her/him to successfully publish in a specific set of topics. The embedding vector space model is then trained to learn the similarity of topics by analyzing the bag-of-topics of all the authors in our dataset.

From a technical standpoint, the model embeds each research topic into a $N$-dimensional space where related research topics are going to be placed close to each other. Each PACS code is thus identified by a vector $v e c_{j}^{t}$ defined by the $N$-dimensional embedding for topic $i$, learned with the StarSpace model [54] by observing scientists publication patterns in time window $t$. In this model, entity embeddings are learned using discrete feature representations describing the relations between the selected entities (in our case, authors and PACS codes). In practice, the model is used in its collaborative filtering-based recommendation training mode where collections of labels-the bag-of-topics for each author-are used to predict/suggest other PACS codes in which an author might be active on. This is achieved by first defining a dictionary of $\mathcal{D}$ features as a $\mathcal{D} \times N$ matrix where the $i$ th row represents the $N$-dimensional PACS code/research topic embedding. In our case, $\mathcal{D}$ is set equal to 854 and it corresponds to the number of PACS codes considered in our analysis; while $N$ is set equal to 200 (this choice is discussed in the Methods section). The embeddings are learned by minimizing a loss function that depends on the pairwise cosine similarities between the different topics. Further details are provided in the Methods section, but the basic intuition behind this approach is that research topics co-occurrences 


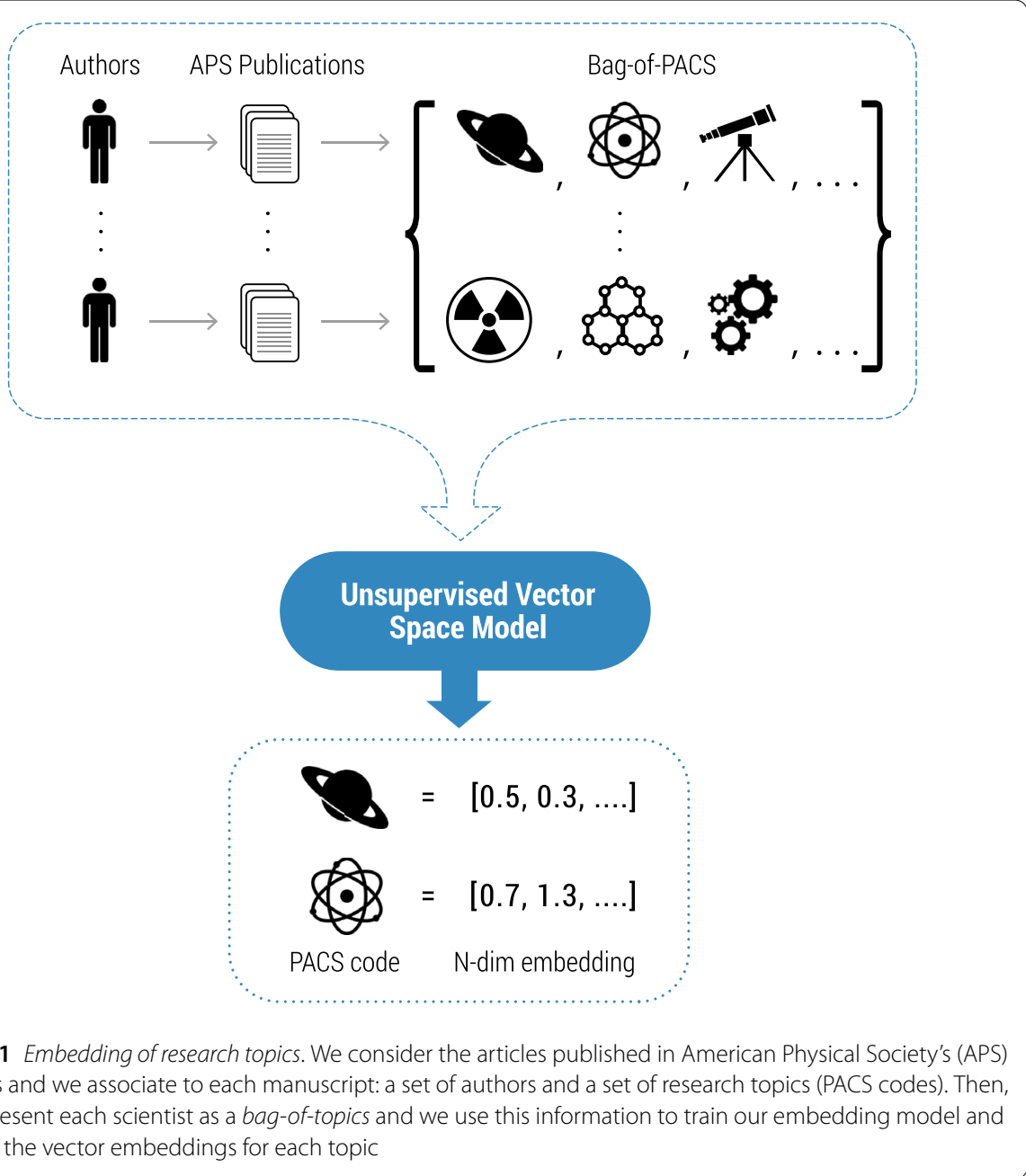

(at the author level) are exploited to tune the embeddings so that frequently occurring pairs-of-topics are also close in the $N$-dimensional embedding space.

\subsection{From the embedding space to the research space network}

The embedding of topics into a high dimensional research space allows us to use their spatial positions to infer the value of their pairwise similarities by measuring the topics relative closeness. In particular, the topic vector space can be used to compute the similarity between two research topics as the cosine similarity between their vectors:

$$
\phi_{i, j}^{t}=\frac{v e c_{i}^{t} \cdot v e c_{j}^{t}}{\left\|v e c_{i}^{t}\right\|\left\|v e c_{j}^{t}\right\|},
$$

where $v e c_{i}^{t}$ and $v e c_{j}^{t}$ are the 200-dimensional embeddings for topics $i$ and $j$, respectively.

The similarity measure can be used to generate the research space network (RSN) that considers the similarity as the weight of the connections and preserves only the most important links by removing the ones associated to negative or small values of the cosine similarity. The resulting RSN is visualized in Fig. 2, where we show that our methodology successfully groups together research topics belonging to the same section as taxon- 


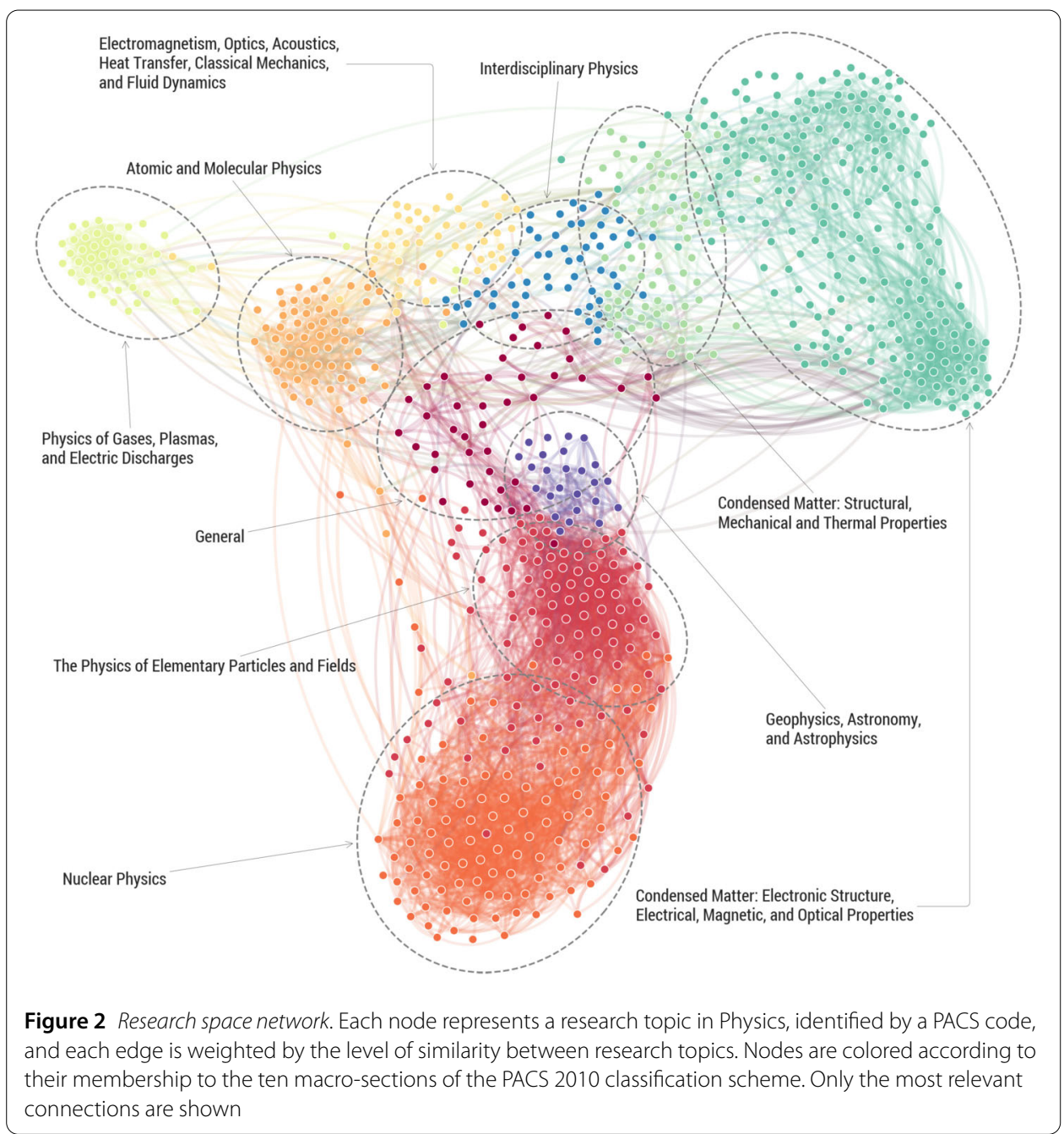

omized in the PACS 2010 Regular Edition of the Physics and Astronomy Classification Scheme [59]. Although our methodology is completely general and does not make use of the PACS hierarchical classification, we can use the latter as an external validation of the quality of the obtained embedding and classification. Indeed, our approach treats each 6-digits PACS code as a mere keyword and the information regarding the hierarchical structure of the classification scheme is not used to train the vector embeddings. In other words, our algorithm is unaware of the existence of the ten Sections of the PACS classification. However, when we do look at the resulting research space by coloring the nodes according to their PACS Section we notice that the General section is correctly placed at the center of the research space network, along with the Interdisciplinary Physics section, as one would expect. On the other hand, we note that Physics of Gases, Plasmas, and Electric Discharges, Condensed Matter, and Nuclear Physics seem to be populating three different boundary areas of the research space (as also observed in previous studies [3]). Overall, the position of the topics is consistent with the information codified in the PACS codes but-in addition - our approach also allows us to understand the relative position of each topic and PACS section with respect to each other, therefore enabling us to quantitavely measure their degree of relatedness. 


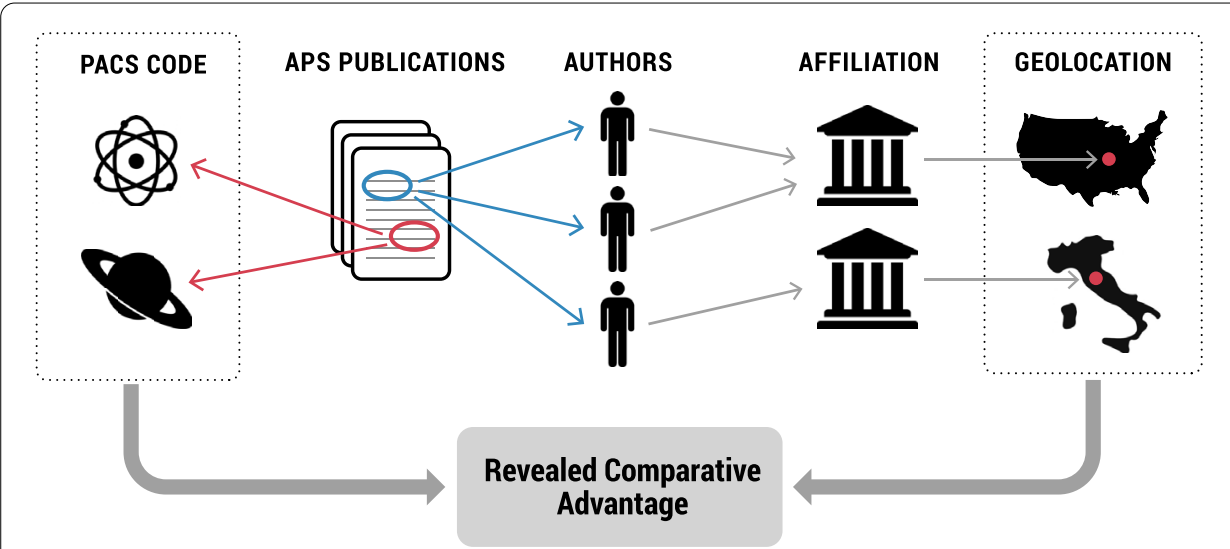

Figure 3 Data representation. Papers are geo-localized using authors' affiliation information, while research topics correspond to the PACS codes reported in each article. For each geographical unit and location pair, the revealed comparative advantage (RCA) is computed to generate location-specific specialization profiles that allow us to fingerprint the structure of the scientific production system of each urban area

\subsection{Fingerprinting scientific expertise}

Research activities in the context of the research space can be analyzed at different geographical scales. More precisely, we can fingerprint scientific production at the level of individual authors, institutions, cities, or countries by geolocating scientific publications (Fig. 3). This can be achieved by considering all the articles published in American Physical Society's (APS) journals in the period 1986-2009 and by associating to each publication: (a) the information contained in the authors' affiliation; and (b) the set of research topics (i.e. the PACS codes) used in the paper. In the following, we focus on geographical units constructed by first parsing the city names from the affiliation strings for each article, and then clustering together neighboring cities to obtain distinct urban areas. More specifically, we follow the same procedure used in [60]: first, we infer the country in which each affiliation-city pair is located; second, for each country, we compute a geographic distance matrix (using Vicenty's formula) connecting each pair of cities; and lastly we use hierarchical clustering to define the different urban areas with the additional constraint that the maximum distance within each cluster has to be less than $50 \mathrm{~km}$. Once we have the geographical units defined, we count how many publications have been produced in each PACS code by each distinct urban area.

In order to provide a specific fingerprinting for the degree of specialization of each geographical unit we extend to scientific production [47] the concept of Revealed Comparative Advantage (RCA, [61]). RCA has a long history in the economic literature where it has been used to study the level of specialization of nations and regions in terms of industrial production, technological production, and trade exports (see for example [62-75]). The RCA [61] is defined as:

$$
R C A_{c, k}^{t}=\frac{X_{c, k}^{t} / \sum_{k} X_{c, k}^{t}}{\sum_{c} X_{c, k}^{t} / \sum_{c, k} X_{c, k}^{t}}
$$

where $X_{c, k}^{t}$ denotes the number of publications produced in urban area $c$ in PACS code $k$ in the time window $t$. In practice, the numerator represents the percentage share of papers published in PACS code $k$ by location $c$; while the denominator represents the 


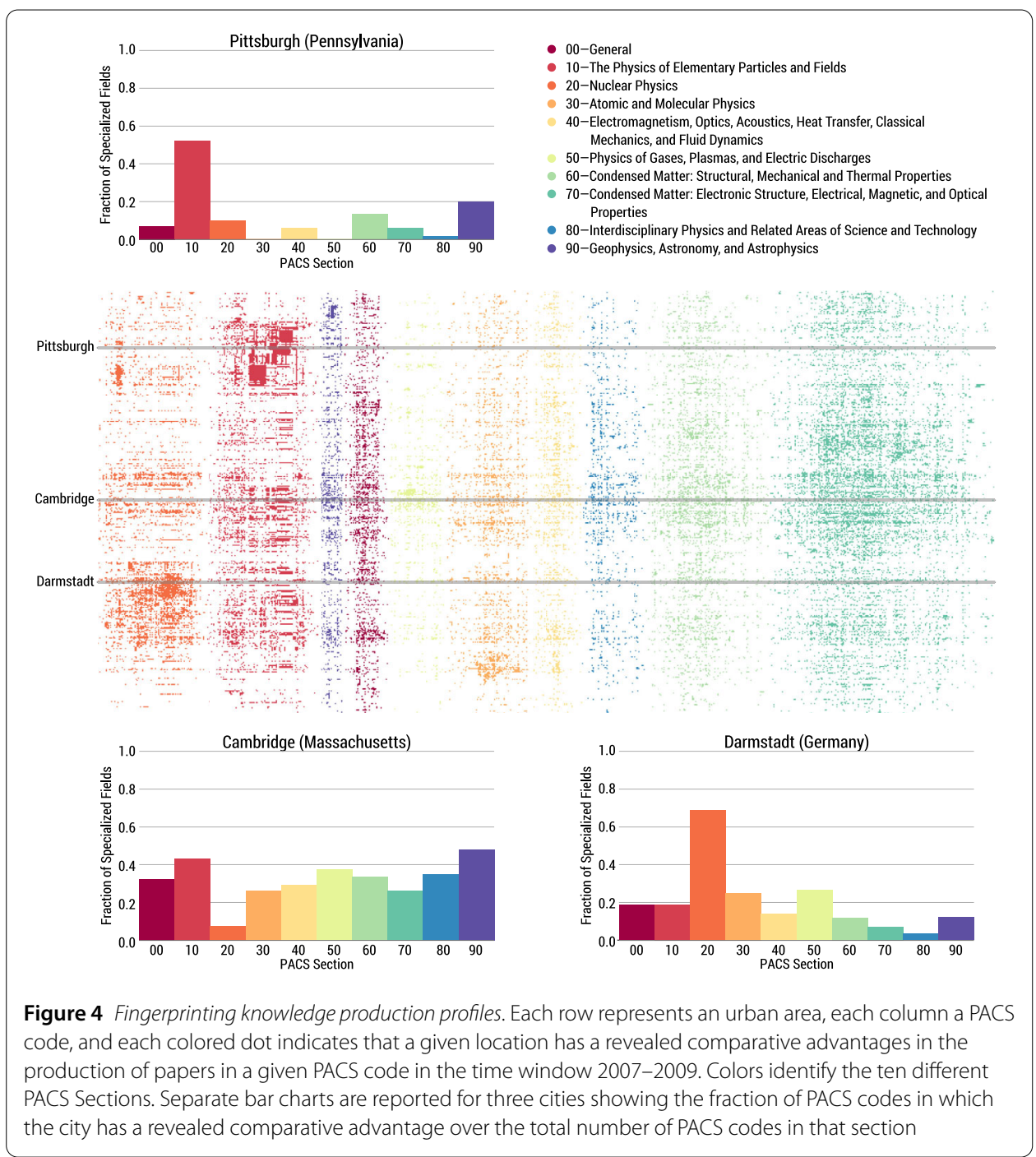

percentage share of papers published in PACS code $k$ across the world. By comparing these two figures, we can assess whether a given geographical unit is relatively more specialized in a certain research topic.

By using the above definition, we consider a geographical unit to be a specialized scientific producer in PACS code $k$ at time $t$ if $R C A_{c, k}^{t}>1$. Using the RCA, we can generate location-specific specialization profiles that allow us to fingerprint the structure of the scientific production system of each geographical area. In particular, we can create a (time varying) fingerprint matrix $F_{c k}$, where $c$ is a geographical unit and $k$ is a topic, and assign non-zero entries only if $R C A_{c, k}^{t}>1$. We can visualize the matrix $F_{c k}$ to have a general understanding of the different specialization patterns, a sort of research DNAs, that characterize the knowledge production of geographical units, as shown in Fig. 4. As an example, we also show the scientific fingerprints of three different urban areas: Darmstadt (Germany), Cambridge (MA, USA), and Pittsburgh (PA, USA). This let us appreciate how different locations might specialize into different parts of the research space. For instance, Darmstadt has a relative comparative advantage on $\sim 70 \%$ of all the PACS code in $\mathrm{Nu}$ clear Physics (PACS section 20). On the other hand, Pittsburgh is specialized in Physics 
of Elementary Particles and Fields (PACS section 10) while its specialization in Nuclear Physics is particularly low. Lastly, we observe that Cambridge is the only city among the three considered with a more homogenous pattern of specialization (with the exception of Nuclear Physics). Overall, by looking at Fig. 4, we can start to appreciate how different cities might cluster their scientific expertise around distinct areas of the research space, even though exceptions - as in the case of Cambridge-do exist.

\subsection{Knowledge density and the prediction of scientific specialization}

The RCA in the context of the research space has been introduced by Guevara et al. [47] to explore the principle of relatedness $[57,58,76]$ in the process of scientific production: i.e. it is easier to specialize and work in related research areas requiring a set of common skills/knowledge. Indeed, relatedness has been found to play an important role in explaining the patterns of future development of industries and research production at the level of cities, regions, and nations $[47,57,77-83]$. This is due to the fact that different sets of capabilities and skills might be needed to grasp the depth and complexity of different research topics, therefore affecting the ability of researchers to move and develop a competitive edge across different disciplines. This observation builds upon the idea that congnitive proximity $[84,85]$ is required to successfully absorb and use new knowledge [86].

Our analysis provides support to the principle of relatedness and the fingerprint matrix shows patterns of specialization that are indeed not random. Some of these patterns can be appreciated in Fig. 5 where we plot each PACS code in which a city has a comparative advantage using the spatial coordinates identified using the research space mapping. Also in this case, we can appreciate how spatial ontologically consistent clusters of competences emerge. In other words, it appears that urban areas tend to develop around their current domain of expertise implying that scientific relatedness does play a role in explaining the structure of knowledge production of a city. In order to quantify the relatedness of a specific PACS code to the overall domain of expertise of a given geographical unit, we use the knowledge density (as proposed in [57]). The knowledge density $\omega_{i, c}^{t}$ around PACS code $i$ in urban area $c$ at time $t$ is defined as:

$$
\omega_{i, c}^{t}=\frac{\sum_{\left\{k \text { s.t. } R C A_{c, k}^{t}>1 \text { and } \phi_{i, k}^{t}>0\right\}} \phi_{i, k}^{t}}{\sum_{\left\{j \text { s.t. } \phi_{i, j}^{t}>0\right\}} \phi_{i, j}^{t}},
$$

where $\phi_{i, j}$ is the level of knowledge similarity between PACS codes $i$ and $j$. In our case, we use cosine similarity to measure the similarity between two research topics. Given this definition, for a location $c$ and time window $t$, the closer topic $i$ is to other topics in which $c$ has a relative comparative advantage, the higher its knowledge density. To understand how this metric works, let us consider what happens when the index-which varies between zero and one-takes its extreme values. For a given PACS code $i$ and urban area $c$ combination, the value of $\omega_{i, c}^{t}$ is equal to zero if $c$ has no comparative advantages in topics related to $i$; while it has a knowledge density equal to one if it has an advantage in all the topics related to $i$. In other words, the closer is $i$ to the current domain of expertise of $c$, the denser the knowledge space will be around PACS code $i$.

For each geographical unit, we can associate the knowledge density $\omega_{i, c}^{t}$ with four different types of transitions that characterize the time evolution of the comparative advantage 


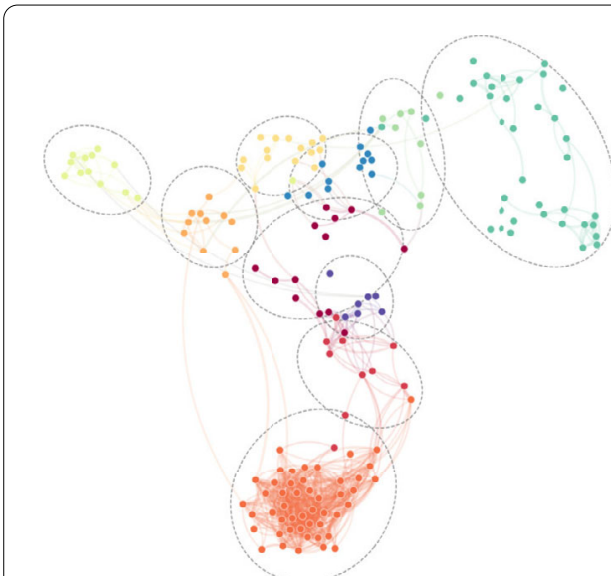

(a) Brussels (Belgium)

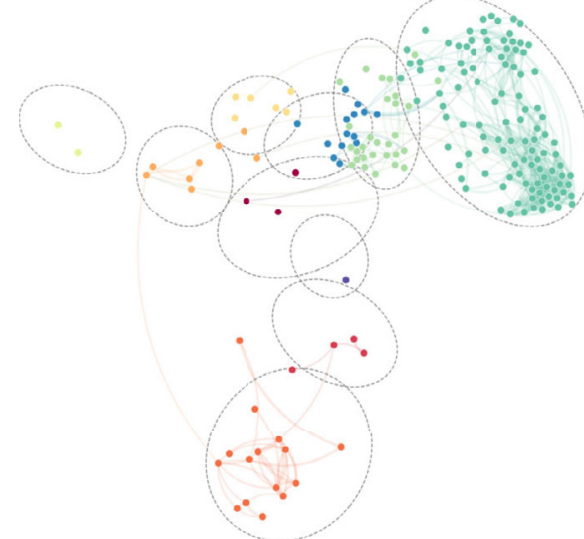

(c) Grenoble (France)

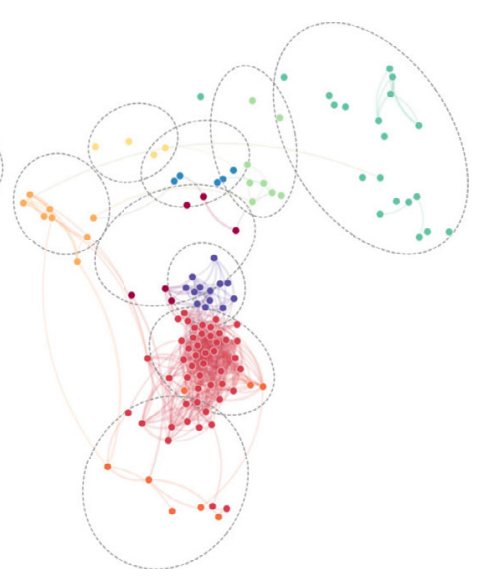

(b) Chicago (Illinois, USA)

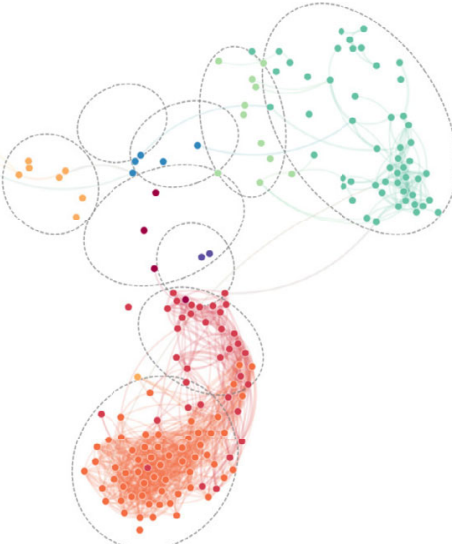

(d) Toyonaka (Japan)

Figure 5 Fingerprints and research space. Specialization in the Physics research space networks of four different cities showing only the PACS codes in which the urban areas exhibit a revealed comparative advantage in the period 2007-2009. Colors identify the ten different PACS sections as in Fig. 4

of a PACS code. We look at the distributions of $\omega_{i, c}^{t}$ when: (1) a PACS code that is inactive (i.e. $R C A=0$ ) at time $t-1$, remains inactive at time $t$; (2) a PACS code that is inactive at time $t-1$ becomes active but with no comparative advantage (i.e. $0<R C A \leq 1$ ) by the city at time $t$ (i.e. inactive to active but not specialized); (3) a PACS code remains active but with no comparative advantage by the city at both $t-1$ and $t$ (i.e. remained not specialized); and lastly (4) a PACS code that is active but with no comparative advantage by the city at time $t-1$, while a comparative advantage (i.e. $R C A>1$ ) emerges at time $t$ (i.e. from not specialized to specialized). Looking at these distributions we observe that PACS codes that normally remain inactive are the ones in which the knowledge density at the previous time step was the lowest, while the opposite holds for the codes in which urban areas become specialized (a visualization of the results is reported in the swarm plot in Fig. 6). In other words, it is easier to develop a stronger comparative advantage in research topics that are related-in the research space-to the ones in which a location is already specialized in.

It is interesting to explore the possibility of using the knowledge density as a predictor of the emergence of a comparative advantage of a city in a specific PACS code in the future. 


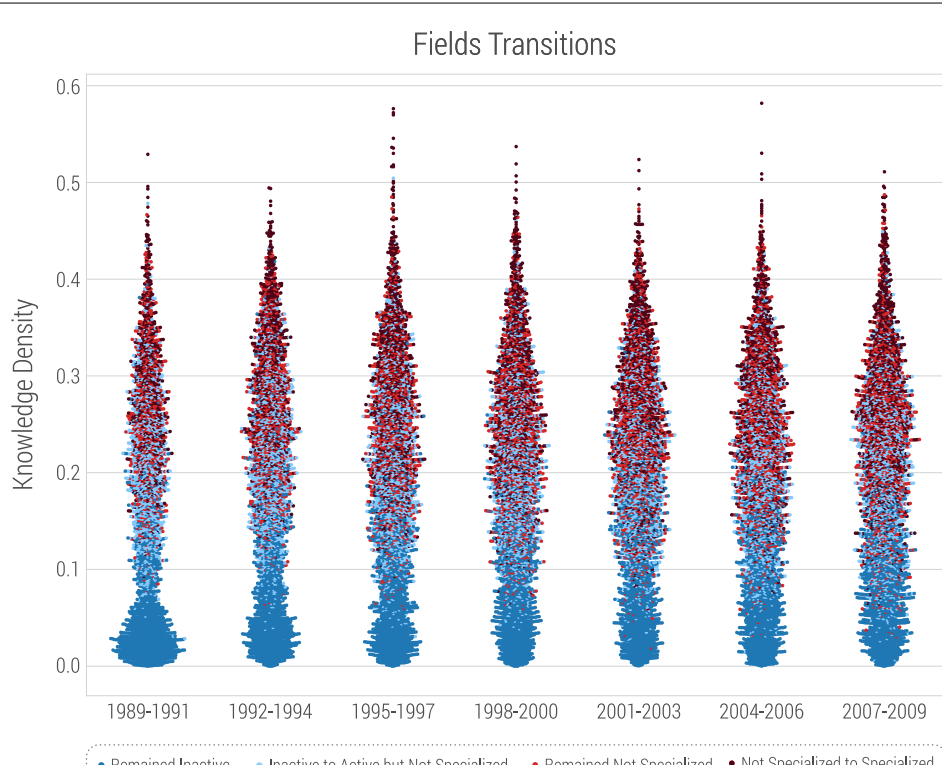

- Remained Inactive • Inactive to Active but Not Specialized • Remained Not Specialized • Not Specialized to Specialized

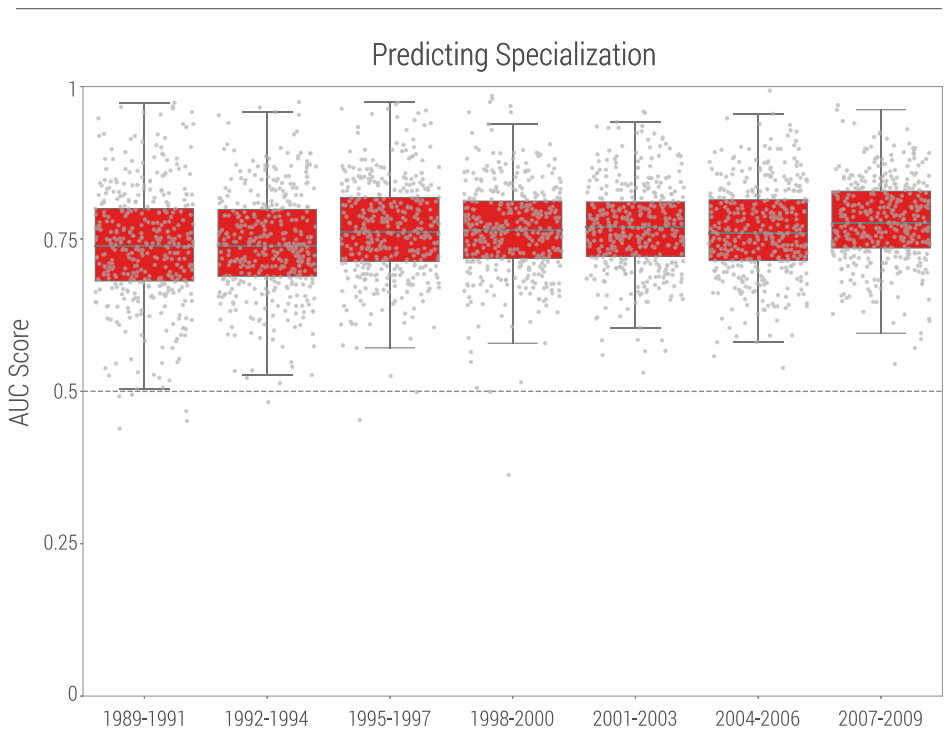

Figure 6 Predicting specialization. Top panel: In the swarm plot every dot represents a PACS code-city pair where the time period is reported on $x$-axis, the observed level of knowledge density on the $y$-axis, and the transition type is identified the color of the dot. From this representation we observe that urban areas tend to develop quicker in activities around which their knowledge density was higher. Bottom panel: The boxplot represents the distribution of the values of the area under the ROC curve when knowledge density is used to predict urban scientific specialization. Values of accuracy greater than $50 \%$ imply a better predictive power than a model assuming random specialization patterns

Operationally, we follow the same methodology proposed in [47] and postulate that the order in which each urban area will become specialized should closely follow the list of PACS codes ranked according to their associated value of knowledge density. We can test this hypothesis against a null one assuming that, instead, specialization occurs independently of the current level of knowledge density. In other words, the alternative hypothesis would suggest that an urban area develops a comparative advantage at random, regardless of its previous level of expertise and specialization. The predictive performance of 
the knowledge density $\omega_{i, c}^{t}$ can be evaluated using a statistic which is normally used in the machine-learning community to measure the accuracy of a model: the area under the so-called Receiver Operating Characteristic (ROC) curve. The ROC curve is used to plot the true negative rate of a model (for example of a classifier) against its true positive rate. That is, the share of correctly classified negative values against the share of correctly classified positive values. If the value of the area under the ROC curve is greater than $50 \%$, then the accuracy of our prediction using the knowledge density is greater than the one we would have from a random prediction where PACS would not have been ranked by their knowledge density. In our case, we actually have a distribution of such values since-for a given time period-we can compute the accuracy of our model for each geographical unit. In other words, we test our ability to predict the research trajectories of each city in each distinct time window. The results, reported in Fig. 6, show that the accuracy is higher than $50 \%$, confirming that the structure of the research space can be used to predict how research trajectories evolve over time. While beyond the scope of the presented work, it is possible to envision the use of current estimate of the knowledge density to forecast the physics research areas in which specific urban areas will be able to specialize in future years.

\section{Discussion}

The construction of the physics research space by embedding topics in a high-dimensional space allows the fingerprinting of the patterns of specialization of urban areas, and the prediction of the evolution of cities' patterns of specialization across different research topics, providing additional support to the principle of relatedness $[57,58,76]$. However, the observed level of scientific capacity, as characterized by the value of the knowledge density, varies considerably in relation to the socio-economic status of each specific geographical areas. To highlight this aspect, we zoom-out and repeat the same exercise we performed for urban areas at the level of countries and we compute the overall average knowledge density of each nation across all PACS sections and for each PACS section. Then, we use the measured average knowledge density to study the association of this measure of national scientific competence with several World Development Indicators (WDI) [87] that quantify the socio-economic status of the countries under analysis. In Fig. 7 we report an example of the associations found for 67 countries. This set of countries represents approximately $99 \%$ of the total publications in our dataset. In Table 1 we show a summary of the results for all WDI considered, and in Fig. 8 we report the average correlation for each indicator category broken down by PACS section. This correlation analysis suggests that the most advanced countries-in terms of scientific expertise in Physics-are also the ones with the higher share of production and export of high-tech goods, the higher levels of investment in R\&D, the higher levels of production of measurable innovation outcomes (e.g. patent, industrial design, and trademark applications), the higher levels of educational attainment and-at the same time-the lower levels of unemployment of skilled labor. Overall, this picture shows that economic development goes hand in hand with a high value of (average) knowledge density, thus supporting the key role of scientific production in the economic growth of nations.

It is worth remarking that the study presented here is considering only the Physics literature published in APS journals, thus missing out on more complex dynamics that could explain the (co)evolution of scientific expertise in different scientific domains in both time 

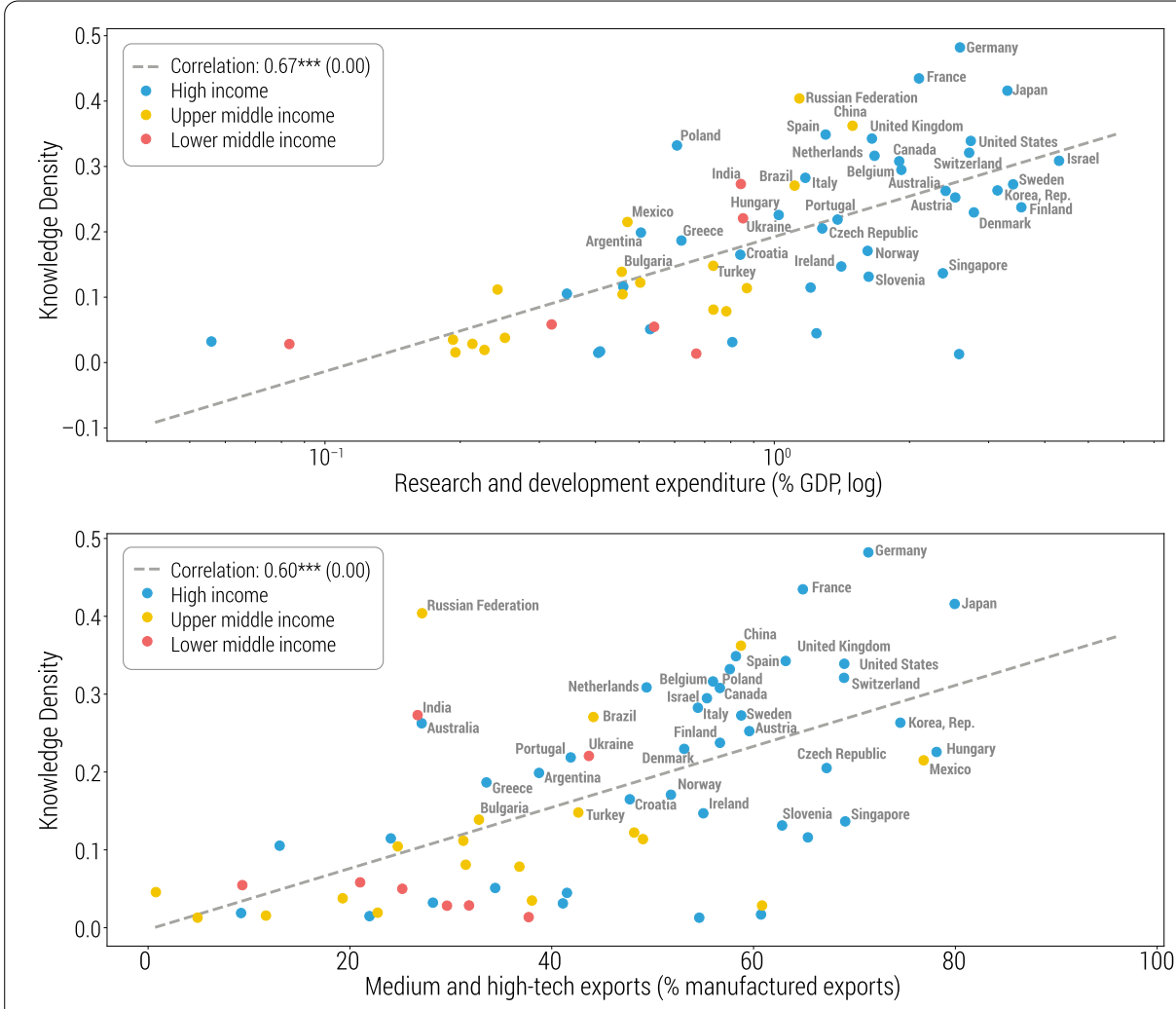

Figure 7 Knowledge density, R\&D, and exports. Relation between the average knowledge density of a country in the last time window of our sample (2007-2009) with its level of expenditure in research and development reported as a fraction of the gross domestic product (GDP) and with its level of trade in medium and high-tech products reported as a fraction of its manufactured exports

and space. Furthermore we relied on the Physics and Astronomy Classification Scheme to assign topics to articles, thus constraining the research space to a pre-defined taxonomy. The PACS scheme was however used for the sake of comparing with previous results in the literature, and the proposed approach does not have to be limited to research in Physics, but it can be extended to other disciplines. In order to overcome the above limitations the embeddings can be produced by simply analyzing the text of paper titles and abstracts without any a-priori knowledge of a scientific topic classification and extending the analysis to databases including a wider range of scientific disciplines, ranging from Physics and Engineering to Economics and Philosophy. The proposed approach might also help address the problem of dealing with the bursty behavior [5] of author-defined keywords. Indeed, even short-lived labels can be put in relation to more stable scientific topics since both sets of keywords will live in the same $N$-dimensional embedding.

Another potential application of the framework presented in this paper concerns the study of how scientific concepts change and move over time across the embedding space. This could provide us with a methodology to study "where science is going", i.e. to understand how scientists or research topics move over time. Indeed, in the NLP literature, some approaches have been proposed to study how word analogies and semantic meaning change over time (see for example $[90,91]$ ). Similar techniques could be used in our context to study the temporal evolution of science. 
Table 1 Knowledge density and world development indicators

\begin{tabular}{|c|c|c|c|}
\hline World development indicator & Correlation & Pvalue & Logs \\
\hline \multicolumn{4}{|l|}{ Economic Development } \\
\hline Economic Fitness Metric $[88,89]$ & $0.76^{* * *}$ & 0.00 & \\
\hline GDP per capita & $0.47^{* * *}$ & 0.00 & $x$ \\
\hline \multicolumn{4}{|l|}{ Research and Development } \\
\hline Research and development expenditure (\% of GDP) & $0.67^{* * *}$ & 0.00 & $x$ \\
\hline Researchers in R\&D (per million people) & $0.52^{* * *}$ & 0.00 & $x$ \\
\hline Technicians in R\&D (per million people) & $0.55^{* * *}$ & 0.00 & $x$ \\
\hline Industrial design applications by residents & $0.72^{* * *}$ & 0.00 & $x$ \\
\hline Industrial design applications by nonresidents & $0.49^{* * *}$ & 0.00 & $x$ \\
\hline Patent applications by residents & $0.82^{* * *}$ & 0.00 & $x$ \\
\hline Patent applications by nonresidents & $0.43^{* * *}$ & 0.00 & $x$ \\
\hline Trademark applications by residents & $0.71^{* * *}$ & 0.00 & $x$ \\
\hline Trademark applications by nonresidents & $0.58^{* * *}$ & 0.00 & $x$ \\
\hline \multicolumn{4}{|l|}{ Production and Exports } \\
\hline $\begin{array}{l}\text { Medium and high-tech Industry } \\
\text { (\% manufacturing value added) }\end{array}$ & $0.55^{* * *}$ & 0.00 & \\
\hline $\begin{array}{l}\text { Medium and high-tech exports } \\
\text { (\% manufactured exports) }\end{array}$ & $0.60^{* * *}$ & 0.00 & \\
\hline High-technology exports & $0.77^{* * *}$ & 0.00 & $x$ \\
\hline \multicolumn{4}{|l|}{ Education Attainment } \\
\hline $\begin{array}{l}\text { At least completed lower secondary } \\
\text { (\% 25+ population) }\end{array}$ & $0.41^{* *}$ & 0.00 & \\
\hline $\begin{array}{l}\text { At least completed upper secondary } \\
(\% 25+\text { population })\end{array}$ & $0.34^{*}$ & 0.02 & \\
\hline $\begin{array}{l}\text { At least completed post-secondary } \\
(\% 25+\text { population })\end{array}$ & 0.25 & 0.08 & \\
\hline $\begin{array}{l}\text { At least completed short-cycle tertiary } \\
\text { (\% 25+ population) }\end{array}$ & $0.32^{*}$ & 0.02 & \\
\hline \multicolumn{4}{|l|}{ Unemployment of Educated Labor Force } \\
\hline $\begin{array}{l}\text { Unemployment with advanced education } \\
\text { (\% of total labor force with advanced education) }\end{array}$ & $-0.34^{*}$ & 0.01 & \\
\hline $\begin{array}{l}\text { Unemployment with intermediate education } \\
\text { (\% of total labor force with intermediate education) }\end{array}$ & -0.22 & 0.10 & \\
\hline \multicolumn{4}{|l|}{ NEET } \\
\hline $\begin{array}{l}\text { Share of youth not in education, employment or } \\
\text { training, female (\% of female youth population) }\end{array}$ & $-0.36^{* *}$ & 0.00 & $x$ \\
\hline $\begin{array}{l}\text { Share of youth not in education, employment or } \\
\text { training, total (\% of youth population) }\end{array}$ & $-0.32^{*}$ & 0.01 & $x$ \\
\hline
\end{tabular}

The generality and scalability of the proposed approach is potentially valuable in the study of the temporal evolution and dynamic of the research space in very large datasets, as well as developing new methodologies for the evaluation of policies, organizations and individuals in the context of consumption and production of knowledge. Furthermore, it has also been shown that word embeddings can be used in machine translation tasks even in absence of multi-language parallel corpora. In other words, monolingual word embeddings can be used to relate words and concepts expressed in different languages without the use of bilingual dictionaries [92, 93]. This result opens the way to study scientific publications produced in any language, without restricting the analysis to English contributions. As a consequence, the impact of scientists and of the role that cities and 


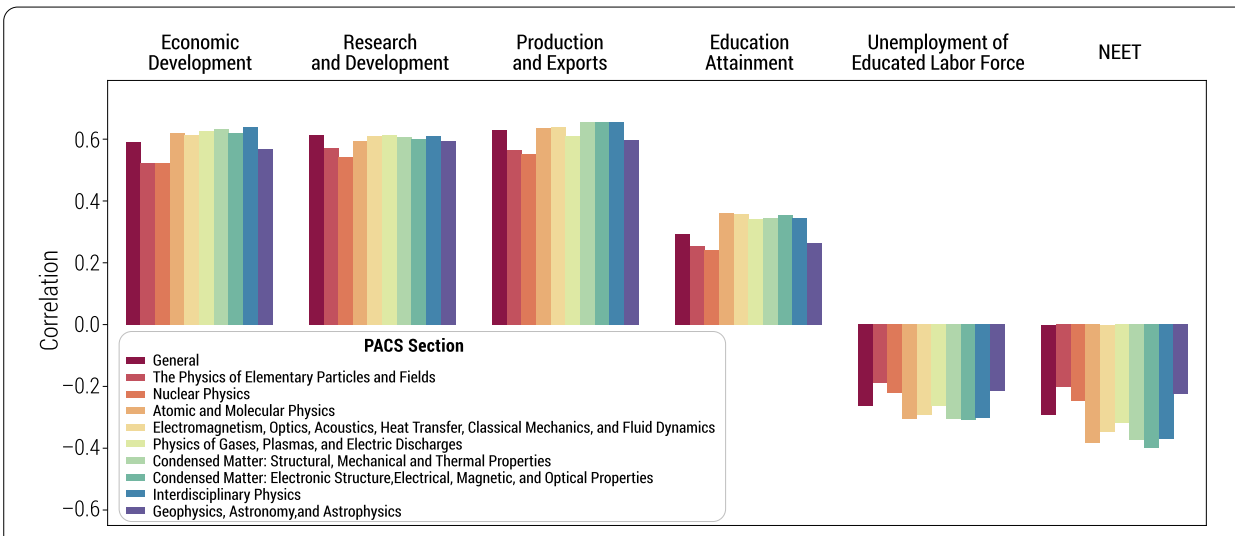

Figure 8 Knowledge density and development indicators. Correlation between the average knowledge density of a country in the period 2007-2009 computed for each one of the ten PACS sections and each development indicator category as introduced in Table 1

countries have in the production of science could be analyzed in a Science Space which could include topics and keywords in any language.

\section{Methods}

\subsection{Data}

In our analysis, we use the APS Data for Research (2010 release) data collection which comprises information about more than 400,000 articles published by the American Physical Society. In particular, in this work, we limit our attention to the years 1986-2009 and we perform our analysis dividing our sample in 3-years non-overlapping time windows. However, the results we provide are robust with respect to the exact choice of the time window size and on whether or not overlapping intervals are considered. Papers are geolocated parsing the information contained in the authors affiliations following the procedure detailed in [60], authors are disambiguated following the procedure detailed in [94], while research topics are assigned considering the first 6 digits of the PACS classification scheme [59]. Overall, our dataset includes 2307 urban areas and 5800+ PACS codes. However, in our analysis, we restrict our attention to cities that have at least 6 publications in each time window. This restricts our original sample to 402 urban areas and 854 PACS codes.

\subsection{Embedding model}

In order to produce the PACS code embeddings, we employ the StarSpace model proposed by [54]. Starspace is a general-purpose embedding model that aims at creating embeddings for a variety of entity types (e.g. words, sentences, documents, images, etc.) by associating to each entity an $N$-dimensional vector. In our case, the vector size is set to $N=200$ and the vectors are obtained by minimizing a loss function that simultaneously maximizes the (cosine) similarity between embeddings of PACS that are used by the same author, and by minimizing the (cosine) similarity between embeddings of PACS that do not appear together when looking at the career of scientists. In other words, once PACS codes are mapped into this new 200-dimensional space, PACS that frequently appear together in the list of publications of a scientist will tend to be close, while PACS that rarely appear together will belong to different areas of the embedding space. More specifically, the model 


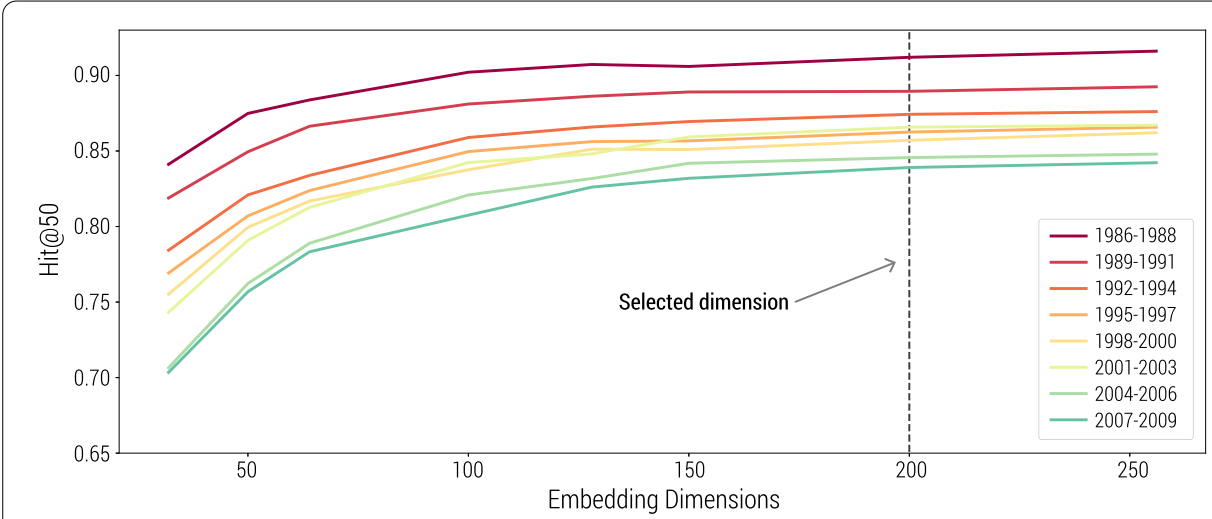

Figure 9 Embedding model performance. Values of the performance metric hits@k [96] for $k=50$. This shows how the performance of the embedding model varies with the size of the embedding dimension $N$

minimizes the following loss function:

$$
\sum_{\substack{(a, b) \in E^{+} \\ b^{-} \in E^{-}}} L^{\text {batch }}\left(\operatorname{sim}(a, b), \operatorname{sim}\left(a, b_{1}^{-}\right), \ldots, \operatorname{sim}\left(a, b_{k}^{-}\right)\right)
$$

where $E^{+}$denotes the set of positive entity pairs (i.e. PACS that often appear together), $E^{-}$denotes the set of negative entity pairs (i.e. PACS that rarely appear together), $\kappa=50$ is the number of negative pairs used for each batch update (i.e., this model uses a $K$ negative sampling strategy as in [50]), $\operatorname{sim}(\cdot)$ denotes the cosine similarity between two embeddings, and $L^{\text {batch }}$ denotes the batch specific loss function that compares the positive pair $(a, b)$ with the negative pairs $\left(a, b_{i}^{-}\right)$using a margin ranking loss of the form $\max \left(0, \mu-\operatorname{sim}(a, b)+\sum_{i \in[1, \kappa]}\left(a, b_{i}^{-}\right)\right)$. The loss function is then minimized using stochastic gradient descent [95]. The value of $N$ has been chosen after examining the prediction performance of our model when trying to reconstruct the bag-of-topics of the authors. In particular, we computed the percentage of correctly predicted PACS codes in the top $k$ predictions made by the algorithm. This metric is commonly denoted by hit@k [96] and it is the same performance metric used also in [54]. In Fig. 9 we show how its value varies with the size of the embedding dimension $N$ and $k=50$. In light of this analysis, we decided to set $N$ equal to 200 since it provided a good compromise between the training time required to fit the model and its overall prediction quality.

\section{Acknowledgements}

This work has been partially supported by Air Force Office of Scientific Research grant FA9550-15-1-0077. We acknowledge the American Physical Society for providing access to the publication data.

\section{Abbreviations}

APS, American Physical Society; PACS, Physics and Astronomy Classification Scheme; RSN, research space network; RCA Revealed Comparative Advantage; ROC, Receiver Operating Characteristic.

\section{Availability of data and materials}

Access to the original APS Data Sets for Research can be requested at the following webpage: https://journals.aps.org/datasets. The source code for StarSpace model [54] can be accessed at https://github.com/facebookresearch/StarSpace. Additional data and codes can be made available upon request to the authors. 


\section{Authors' contributions}

$M C$ and $A V$ designed research; $M C, B G, Q Z$ performed research; $M C, B G$, $A V$, and $Q Z$ analyzed data; and $M C, B G$, $A V$, and $Q Z$ wrote the paper. All authors read and approved the final manuscript.

\section{Author details}

'MOBS Lab, Northeastern University, Boston, USA. ${ }^{2}$ Institute for Scientific Interchange Foundation, Torino, Italy.

\section{Publisher's Note}

Springer Nature remains neutral with regard to jurisdictional claims in published maps and institutional affiliations.

Received: 21 November 2018 Accepted: 30 September 2019 Published online: 06 November 2019

\section{References}

1. Zeng A, Shen Z, Zhou J, Wu J, Fan Y, Wang Y, Stanley HE (2017) The science of science: from the perspective of complex systems. Phys Rep 714-715:1-73

2. Fortunato S, Bergstrom CT, Börner K, Evans JA, Helbing D, Milojević S, Petersen AM, Radicchi F, Sinatra R, Uzzi B et al (2018) Science of science. Science 359(6379):0185

3. Sinatra R, Deville P, Szell M, Wang D, Barabási A-L (2015) A century of physics. Nat Phys 11(10):791

4. Battiston F, Musciotto F, Wang D, Barabási A-L, Szell M, Sinatra R (2019) Taking census of physics. Nat Rev Phys 1(1):89

5. Hoonlor A, Szymanski BK, Zaki MJ (2013) Trends in computer science research. Commun ACM 56(10):74-83

6. Cheng Q, Lu X, Liu Z, Huang J (2015) Mining research trends with anomaly detection models: the case of social computing research. Scientometrics 103(2):453-469

7. Effendy S, Yap RHC (2017) Analysing trends in computer science research: a preliminary study using the Microsoft academic graph. In: WWW'17 companion proceedings of the 26th international conference on World Wide Web companion, pp 1245-1250

8. Della Briotta Parolo P, Pan RK, Ghosh R, Huberman BA, Kaski K, Fortunato S (2015) Attention decay in science. J Informetr 9(4):734-745

9. Yin Y, Wang D (2017) The time dimension of science: connecting the past to the future. J Informetr 11(2):608-621

10. Pan RK, Petersen AM, Pammolli F, Fortunato S (2018) The memory of science: inflation, myopia, and the knowledge network. J Informetr 12(3):656-678

11. Wagner CS, Roessner JD, Bobb K, Klein JT, Boyack KW, Keyton J, Rafols I, Börner K (2011) Approaches to understanding and measuring interdisciplinary scientific research (IDR): a review of the literature. J Informetr 5(1):14-26

12. Leydesdorff L, Rafols I (2011) Indicators of the interdisciplinarity of journals: diversity, centrality, and citations. J Informetr 5(1):87-100

13. Bromham L, Dinnage R, Hua XX (2016) Interdisciplinary research has consistently lower funding success. Nature 534(7609):684-687

14. Leydesdorff $L$, Wagner CS, Bornmann $L$ (2019) Interdisciplinarity as diversity in citation patterns among journals: Rao-Stirling diversity, relative variety, and the Gini coefficient. J Informetr 13(1):255-269

15. Franzoni C, Scellato G, Stephan $P$ (2014) The mover's advantage: the superior performance of migrant scientists. Econ Lett 122(1):89-93

16. Deville P, Wang D, Sinatra R, Song C, Blondel VD, Barabási AL (2015) Career on the move: geography, stratification, and scientific impact. Sci Rep 4(1):4770

17. Scellato G, Franzoni C, Stephan PE (2015) Migrant scientists and international networks. Res Policy 44(1):108-120

18. Foster JG, Rzhetsky A, Evans JA (2015) Tradition and innovation in scientists' research strategies. Am Sociol Rev 80(5):875-908

19. Jia T, Wang D, Szymanski BK (2017) Quantifying patterns of research-interest evolution. Nat Hum Behav 1(4):78

20. Arrieta OAD, Pammolli F, Petersen AM (2017) Quantifying the negative impact of brain drain on the integration of European science. Sci Adv 3(4):e1602232

21. Vaccario G, Verginer L, Schweitzer F (2018) Reproducing scientists' mobility: a data-driven model. arXiv preprint. arXiv:1811.07229

22. James C, Pappalardo L, Sirbu A, Simini F (2018) Prediction of next career moves from scientific profiles. arXiv preprint. arXiv:1802.04830

23. Wuchty S, Jones BF, Uzzi B (2007) The increasing dominance of teams in production of knowledge. Science 316(5827):1036-1039

24. Bettencourt LMA, Kaiser DI, Kaur J (2009) Scientific discovery and topological transitions in collaboration networks. J Informetr 3(3):210-221

25. Milojević S (2014) Principles of scientific research team formation and evolution. Proc Natl Acad Sci USA 111(11):3984-3989

26. Petersen AM (2015) Quantifying the impact of weak, strong, and super ties in scientific careers. Proc Natl Acad Sci USA 112(34):201501444

27. Larivière $V$, Gingras $Y$, Sugimoto $C R$, Tsou A (2015) Team size matters: collaboration and scientific impact since 1900 J Assoc Inf Sci Technol 66(7):1323-1332

28. Zeng XHT, Duch J, Sales-Pardo M, Moreira JAG, Radicchi F, Ribeiro HV, Woodruff TK, Amaral LAN (2016) Differences in collaboration patterns across discipline, career stage, and gender. PLoS Biol 14(11):e1002573

29. Czaika M, Orazbayev S (2018) The globalisation of scientific mobility, 1970-2014. Appl Geogr 96:1-10

30. Hall KL, Vogel AL, Huang GC, Serrano KJ, Rice EL, Tsakraklides SP, Fiore SM (2018) The science of team science: a review of the empirical evidence and research gaps on collaboration in science. Am Psychol 73(4):532-548

31. Bu Y, Murray DS, Ding Y, Huang Y, Zhao Y (2018) Measuring the stability of scientific collaboration. Scientometrics 114(2):463-479

32. Abramo G, D'Angelo CA, Costa FD (2019) The collaboration behavior of top scientists. Scientometrics 118(1):215-232

33. Yu S, Bedru HD, Lee I, Xia F (2019) Science of scientific team science: a survey. Comput Sci Rev 31:72-83 
34. Wagner CS, Whetsell TA, Mukherjee S (2019) International research collaboration: novelty, conventionality, and atypicality in knowledge recombination. Res Policy 48(5):1260-1270

35. Petersen AM, Riccaboni M, Stanley HE, Pammolli F (2012) Persistence and uncertainty in the academic career. Proc Natl Acad Sci USA 109(14):5213-5218

36. Penner O, Pan RK, Petersen AM, Kaski K, Fortunato S (2013) On the predictability of future impact in science. Sci Rep 3(1):3052

37. Petersen AM, Fortunato S, Pan RK, Kaski K, Penner OB, Rungi A, Riccaboni M, Stanley HE, Pammolli F (2014) Reputation and impact in academic careers. Proc Natl Acad Sci USA 111(43):15316-15321

38. Wang D, Song C, Barabási A-L (2013) Quantifying long-term scientific impact. Science 342(6154):127-132

39. Sinatra R, Wang D, Deville P, Song C, Barabási A-L (2016) Quantifying the evolution of individual scientific impact. Science 354(6312):5239

40. Clauset A, Larremore DB, Sinatra R (2017) Data-driven predictions in the science of science. Science 355(6324):477-480

41. Veugelers R, Wang J (2019) Scientific novelty and technological impact. Res Policy 48(6):1362-1372

42. Börner K, Chen C, Boyack KW (2003) Visualizing knowledge domains. Annu Rev Inf Sci Technol 37(1):179-255

43. Boyack KW, Klavans R, Börner K (2005) Mapping the backbone of science. Scientometrics 64(3):351-374

44. Leydesdorff L, Rafols I (2009) A global map of science based on the isi subject categories. J Am Soc Inf Sci Technol 60(2):348-362

45. Rafols I, Porter AL, Leydesdorff L (2010) Science overlay maps: a new tool for research policy and library management. J Am Soc Inf Sci Technol 61(9):1871-1887

46. Bollen J, Van de Sompel H, Hagberg A, Bettencourt L, Chute R, Rodriguez MA, Balakireva L (2009) Clickstream data yields high-resolution maps of science. PLoS ONE 4(3):4803

47. Guevara MR, Hartmann D, Aristarán M, Mendoza M, Hidalgo CA (2016) The research space: using career paths to predict the evolution of the research output of individuals, institutions, and nations. Scientometrics 109(3):1695-1709

48. Bengio Y, Ducharme R, Vincent P, Jauvin C (2003) A neural probabilistic language model. J Mach Learn Res 3:1137-1155

49. Mikolov T, Sutskever I, Chen K, Corrado GS, Dean J (2013) Distributed representations of words and phrases and their compositionality. In: Burges CJC, Bottou L, Welling M, Ghahramani Z, Weinberger KQ (eds) Advances in neural information processing systems, vol 26. Curran Associates, Inc., New York, pp 3111-3119

50. Mikolov T, Chen K, Corrado G, Dean J (2013) Efficient estimation of word representations in vector space. arXiv preprint. arXiv:1301.3781

51. Huang EH, Socher R, Manning CD, Ng AY (2012) Improving word representations via global context and multiple word prototypes. In: Proceedings of the 50th annual meeting of the association for computational linguistics: long papers, vol 1. Association for Computational Linguistics, Stroudsburg, pp 873-882

52. Pennington J, Socher R, Manning C (2014) Glove: global vectors for word representation. In: Proceedings of the 2014 conference on empirical methods in natural language processing (EMNLP). pp 1532-1543

53. Bojanowski P, Grave E, Joulin A, Mikolov T (2017) Enriching word vectors with subword information. Trans Assoc Comput Linguist 5:135-146

54. Wu L, Fisch A, Chopra S, Adams K, Bordes A, Weston J (2017) Starspace: embed all the things! arXiv preprint. arXiv:1709.03856

55. Salton G, Wong A, Yang C-S (1975) A vector space model for automatic indexing. Commun ACM 18(11):613-620

56. Small H (1973) Co-citation in the scientific literature: a new measure of the relationship between two documents. J Am Soc Inf Sci 24(4):265-269

57. Hidalgo CA, Klinger B, Barabási A-L, Hausmann R (2007) The product space conditions the development of nations. Science 317(5837):482-487

58. Hidalgo CA, Balland P-A, Boschma R, Delgado M, Feldman M, Frenken K, Glaeser E, He C, Kogler DF, Morrison A et al (2018) The principle of relatedness. In: International conference on complex systems. Springer, Cham, pp 451-457

59. AlP-Publishing: PACS 2010 Regular Edition (2010). https://publishing.aip.org/publishing/pacs/pacs-2010-regular-edition. Accessed 2017-08-03

60. Zhang Q, Perra N, Gonçalves B, Ciulla F, Vespignani A (2013) Characterizing scientific production and consumption in physics. Sci Rep 3:1640

61. Balassa B (1965) Trade liberalisation and "revealed" comparative advantage. Manch Sch 33(2):99-123

62. Aquino A (1981) Changes over time in the pattern of comparative advantage in manufactured goods: an empirical analysis for the period 1962-1974. Eur Econ Rev 15(1):41-62

63. Soete LG, Wyatt SM (1983) The use of foreign patenting as an internationally comparable science and technology output indicator. Scientometrics 5(1):31-54

64. Crafts NF, Thomas M (1986) Comparative advantage in uk manufacturing trade, 1910-1935. Econ J 96(383):629-645

65. Van Hulst N, Mulder R, Soete LL (1991) Exports and technology in manufacturing industry. Weltwirtsch Arch 127(2):246-264

66. Cantwell J (1995) The globalisation of technology: what remains of the product cycle model? Camb J Econ 19(1):155-174

67. Amiti M (1999) Specialization patterns in Europe. Weltwirtsch Arch 135(4):573-593

68. lapadre PL (2001) Measuring international specialization. Int Adv Econ Res 7(2):173-183

69. De Benedictis L, Gallegati M, Tamberi M (2008) Semiparametric analysis of the specialization-income relationship. Appl Econ Lett 15(4):301-306

70. OECD (2011) Globalisation, comparative advantage and the changing dynamics of trade. OECD Publishing, Paris

71. Amighini A, Leone M, Rabellotti R (2011) Persistence versus change in the international specialization pattern of Italy: how much does the 'district effect' matter? Reg Stud 45(3):381-401

72. D'Agostino LM, Laursen K, Santangelo GD (2013) The impact of R\&D offshoring on the home knowledge production of OECD investing regions. J Econ Geogr 13(1):145-175

73. Liegsalz J, Wagner S (2013) Patent examination at the state intellectual property office in China. Res Policy 42(2):552-563 
74. Bahar D, Hausmann R, Hidalgo CA (2014) Neighbors and the evolution of the comparative advantage of nations: evidence of international knowledge diffusion? I Int Econ 92(1):111-123

75. Freund C, Pierola MD (2015) Export superstars. Rev Econ Stat 97(5):1023-1032

76. Boschma R, Frenken K, Bathelt H, Feldman M, Kogler D et al (2012) Technological relatedness and regional branching In: Beyond territory. Dynamic geographies of knowledge creation, diffusion and innovation, pp 64-68

77. Neffke F, Henning M, Boschma R (2011) How do regions diversify over time? Industry relatedness and the development of new growth paths in regions. Econ Geogr 87(3):237-265

78. Kogler DF, Rigby DL, Tucker I (2013) Mapping knowledge space and technological relatedness in US cities. Eur Plan Stud 21(9):1374-1391

79. Boschma R, Minondo A, Navarro M (2013) The emergence of new industries at the regional level in Spain: a proximity approach based on product relatedness. Econ Geogr 89(1):29-51

80. Boschma R, Heimeriks G, Balland P-A (2014) Scientific knowledge dynamics and relatedness in biotech cities. Res Policy 43(1):107-114

81. Boschma R, Balland P-A, Kogler DF (2014) Relatedness and technological change in cities: the rise and fall of technological knowledge in us metropolitan areas from 1981 to 2010. Ind Corp Change 24(1):223-250

82. Essletzbichler J (2015) Relatedness, industrial branching and technological cohesion in us metropolitan areas. Reg Stud 49(5):752-766

83. Rigby DL (2015) Technological relatedness and knowledge space: entry and exit of us cities from patent classes. Reg Stud 49(11):1922-1937

84. Boschma R (2005) Proximity and innovation: a critical assessment. Reg Stud 39(1):61-74

85. Boschma R, Frenken K (2010) The spatial evolution of innovation networks: a proximity perspective. In: The handbook of evolutionary economic geography. Edward Elgar, Cheltenham Glos

86. Cohen WM, Levinthal DA (2000) Absorptive capacity: a new perspective on learning and innovation. In: Strategic learning in a knowledge economy. Elsevier, Amsterdam, pp 39-67

87. World Bank: world development indicators (2019). http://datatopics.worldbank.org/world-development-indicators/. Accessed 2019-04-04

88. Tacchella A, Cristelli M, Caldarelli G, Gabrielli A, Pietronero L (2012) A new metrics for countries' fitness and products' complexity. Sci Rep 2:723

89. Cristelli M, Tacchella A, Cader M, Roster K, Pietronero L (2017) On the predictability of growth. Policy Research working paper (WPS8117)

90. Hamilton WL, Leskovec J, Jurafsky D (2016) Diachronic word embeddings reveal statistical laws of semantic change. arXiv preprint. arXiv:1605.09096

91. Szymanski T (2017) Temporal word analogies: identifying lexical replacement with diachronic word embeddings. In: Proceedings of the 55th annual meeting of the association for computational linguistics (volume 2: short papers), vol 2, pp 448-453

92. Conneau A, Lample G, Ranzato M, Denoyer L, Jégou H (2017) Word translation without parallel data. arXiv preprint. arXiv:1710.04087

93. Lample G, Conneau A, Denoyer L, Ranzato M (2017) Unsupervised machine translation using monolingual corpora only. arXiv preprint. arXiv: 1711.00043

94. Radicchi F, Fortunato S, Markines B, Vespignani A (2009) Diffusion of scientific credits and the ranking of scientists, Phys Rev E 80(5):056103

95. Duchi J, Hazan E, Singer Y (2011) Adaptive subgradient methods for online learning and stochastic optimization. J Mach Learn Res 12:2121-2159

96. Frome A, Corrado GS, Shlens J, Bengio S, Dean J, Ranzato M, Mikolov T (2013) Devise: a deep visual-semantic embedding model. In: Advances in neural information processing systems, vol 26, pp 2121-2129

\section{Submit your manuscript to a SpringerOpen ${ }^{\circ}$ journal and benefit from:}

- Convenient online submission

- Rigorous peer review

- Open access: articles freely available online

- High visibility within the field

- Retaining the copyright to your article

Submit your next manuscript at $\gg$ springeropen.com 